\title{
Emergence and spread of predominantly community- onset Clostridium difficile PCR ribotype 244 infection in Australia, 2010 to 2012
}

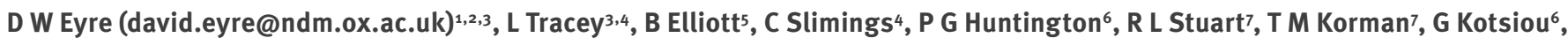

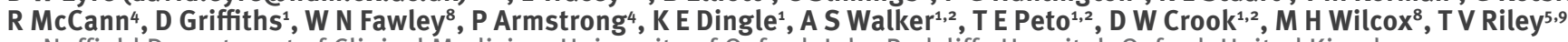

1. Nuffield Department of Clinical Medicine, University of Oxford, John Radcliffe Hospital, Oxford, United Kingdom

2. National Institute for Health Research Oxford Biomedical Research Centre, John Radcliffe Hospital, Oxford, United Kingdom

3. These authors contributed equally to the work

4. Communicable Diseases Control Directorate, Health Department of Western Australia, Shenton Park, Australia

5. Microbiology and Immunology, The University of Western Australia, Nedlands, Australia

6. Microbiology, Pathology North, Royal North Shore Hospital, St Leonards, Australia

7. Monash Infectious Diseases and Monash University, Clayton, Australia

8. Department of Microbiology, Old Medical School, Leeds Teaching Hospitals NHS Trust and University of Leeds, Leeds General Infirmary, Leeds, United Kingdom

9. Microbiology and Infectious Diseases, PathWest Laboratory Medicine, Nedlands, Australia

Citation style for this article:

Eyre DW, Tracey L, Elliott B, Slimings C, Huntington PG, Stuart RL, Korman TM, Kotsiou G, McCann R, Griffiths D, Fawley WN, Armstrong P, Dingle KE, Walker AS, Peto TE, Crook DW, Wilcox MH, Riley TV. Emergence and spread of predominantly community-onset Clostridium difficile PCR ribotype 244 infection in Australia, 2010 to 2012. Euro Surveill. 2015;20(10): pii=21059. Available online: http://www.eurosurveillance.org/ViewArticle.aspx?Articleld=21059

We describe an Australia-wide Clostridium difficile outbreak in 2011 and 2012 involving the previously uncommon ribotype 244. In Western Australia, 14 of 25 cases were community-associated, 11 were detected in patients younger than 65 years, 14 presented to emergency/outpatient departments, and 14 to nontertiary/community hospitals. Using whole genome sequencing, we confirm ribotype 244 is from the same $C$. difficile clade as the epidemic ribotype 027 . Like ribotype 027 , it produces toxins $A, B$, and binary toxin, however it is fluoroquinolone-susceptible and thousands of single nucleotide variants distinct from ribotype 027. Fifteen outbreak isolates from across Australia were sequenced. Despite their geographic separation, all were genetically highly related without evidence of geographic clustering, consistent with a point source, for example affecting the national food chain. Comparison with reference laboratory strains revealed the outbreak clone shared a common ancestor with isolates from the United States and United Kingdom (UK). A strain obtained in the UK was phylogenetically related to our outbreak. Follow-up of that case revealed the patient had recently returned from Australia. Our data demonstrate new $C$. difficile strains are an on-going threat, with potential for rapid spread. Active surveillance is needed to identify and control emerging lineages.

\section{Introduction}

Clostridium difficile is the most common cause of infectious diarrhoea in hospitalised patients [1], in the United States (US) costing more than USD 3 billion (EUR 2.8 billion) annually [2]. Infection occurs following ingestion of $C$. difficile. Exposure to agents that alter the gut microflora, often antibiotics, [3] is normally required, but about half of the cases with community-onset $C$. difficile infection (CDI) may not have received recent antibiotics [4]. Since 2003, rates of healthcare-related CDI have escalated worldwide, with a new hyper-virulent strain of $C$. difficile (PCR ribotype 027) responsible for outbreaks of severe disease in North America and Europe [5]. Following the first detection of ribotype 027 in Australia in 2008 [6], there have been two known clusters of ribotype 027 infection that occurred in Melbourne and Sydney in 2010 [7]. However, the strain has not become endemic in Australia; it is unclear whether this is because of early recognition and subsequent prevention of large scale spread and/or because of relatively conservative antimicrobial prescribing policies, for example low use of fluoroquinolones to which ribotype 027 is resistant $[5,8]$. Rates of community-associated CDI have also increased in North America and Europe $[9,10]$ and community-associated CDI is estimated to be responsible for more than one third of all CDI cases. Patients with community-associated CDI tend to be younger, less likely to have been exposed to antibiotics, and have fewer co-morbidities than patients with healthcareacquired infection [11].

Australian States and Territories currently operate separate, primarily hospital-based, mandatory surveillance for CDI as part of hospital accreditation. All Australian states have seen a significant increase in hospital-identified CDI incidence since mid-2011, which is unlikely to be completely explained by recent changes to more sensitive molecular diagnostic methods or increased awareness of CDI and testing. The 
proportion of recent CDI cases defined as communityassociated has also increased throughout Australia [12]. A recent report on CDI in Tasmania concluded that the observed increase in CDI was most likely due to acquisition in the community [13].

In October and November 2011, a large cluster of an apparently new (to Australia) ribotype was identified by a reference laboratory in Western Australia (WA) in isolates from New South Wales (NSW). At about the same time, reports emerged from Victoria of cases of CDI initially identified as putative ribotype 027 with the GeneXpert system (Cepheid, Sunnyvale, CA), but actually matching the NSW ribotype [14]. Subsequent interrogation of the WA laboratory database identified further CDIs due to the same ribotype (eventually identified as ribotype 244).

Investigation of the 12 ribotype 244 cases in Victoria, demonstrated that the strain was associated with more severe disease, $58 \%$ had severe disease compared to $25 \%$ of cases with non-ribotype 244 , non-ribotype-027 strains, and increased mortality, $42 \% 30$-day mortality vs $0 \%$ [14]. Whole genome sequencing of one of the 12 ribotype 244 isolates showed it to be from the same clade as ribotype 027, but genetically distinct from ribotype 027 [14]. Ribotype 244 cases have also been identified in New Zealand: In a case-control study, 10 ribotype $244 \mathrm{CDI}$ cases were more likely to develop severe colitis than 20 age- and sex-matched controls with CDI with other ribotypes [15].

In this report, we describe the secular trends in the prevalence of hospital-identified CDI in WA from 2010 to 2012 , and in particular CDI due to ribotype 244 . We use whole genome sequencing of ribotype 244 isolates recovered in WA and two other states to investigate strain clonality. Lastly, we report international spread of this ribotype.

\section{Methods}

\section{Epidemiology}

CDI incidence data were obtained from the WA hospital CDI surveillance programme from January 2010 to December 2012 inclusive. In WA, surveillance for CDI has been mandatory since January 2010 for all public metropolitan, regional and integrated district hospitals, and private hospitals funded to provide care to public patients. These hospitals were also encouraged to submit all $C$. difficile-positive faecal samples for PCR ribotyping from January 2010 onwards, with increasing numbers participating during 2010 and 2011, such that all hospitals were providing samples by October 2011.

A CDI case was defined as a case of diarrhoea, i.e. unformed stool taking the shape of its container, meeting the following criteria: the stool sample yielded a positive result in a laboratory assay for $C$. difficile toxin $\mathrm{A}$ and/or $\mathrm{B}$, or a toxin-producing strain of $\mathrm{C}$. difficile was detected in the stool sample by culture or other means. Cases were only included once in an eightweek period; repeat samples from the same patient after eight weeks were considered a new infection. Patients younger than two years were excluded.

CDI was reported from patients attending any area of a hospital, i.e. all inpatient wards and units (including psychiatric, rehabilitation and aged care admissions) and emergency and outpatient departments (including haemodialysis and day surgery units); as such, reporting reflected the total burden of CDI on a hospital and sampled the surrounding community, as the reported cases included disease with both healthcare- and community onset. Data on recent healthcare facility exposure were available on cases identified at metropolitan hospitals (accounting for the majority of cases), allowing these CDI cases to be classified according to the place of probable exposure as described by Kuijper et al. [16] and recent US guidelines [17]: CDI was classified by location of onset, as healthcare facility (HCF) onset or as community-onset, and by the timing relative to any previous healthcare exposure. CDI onsetmore than 48 hours after HCF admission and within four weeks of discharge was denoted HCF-associated, onset between four and 12 weeks post-discharge was denoted indeterminate/unknown (whether communityonset or HCF-onset), and onset more than 12 weeks following last HCF exposure was denoted communityassociated. HCF-associated CDI rates are reported per 10,000 bed-days. As identified community-associated cases were only a subset of all community-associated cases (cases presenting to primary care facilities and smaller hospitals were not included), simple counts of community-associated cases per quarter are presented. Severe CDI was defined as an episode of CDI with one or more signs of severe colitis [18].

\section{PCR ribotyping and toxin gene profiling}

Crude bacterial template DNA for toxin profiling was prepared by resuspension of cells in a $5 \%$ (wt/vol) solution of Chelex-100 resin (Sigma-Aldrich, Castle Hill, NSW, Australia). All isolates were screened by PCR for the presence of the toxin $A(\operatorname{tcd} A)$ and toxin $B$ $(t c d B)$ genes [19] and the binary toxin ( $c d t A$ and $c d t B$ ) genes [20], and for changes in the repeating region of tcdA [21]. PCR ribotyping was performed as previously described [22]. PCR ribotyping banding patterns were identified by comparison with a reference library consisting of reference strains from the European Centre for Disease Prevention and Control (ECDC)-Brazier collection [23], a collection of the most prevalent PCR ribotypes currently circulating in Australia (B. Elliott, unpublished data), and a selection of binary toxinpositive strains. Ribotyping results were confirmed by the Reference Laboratory of the Clostridium difficile Ribotyping Network (CDRN) for England in Leeds, United Kingdom (UK). Antimicrobial susceptibility testing and breakpoints for metronidazole, vancomycin, clindamycin and moxifloxacin were determined by Clinical and Laboratory Standards Institute agar dilution [24]. 
Whole genome sequencing

Fifteen ribotype 244 isolates obtained between 16 July 2011 and 18 January 2012 and submitted to PathWest for ribotyping at the time of the study were selected for whole genome sequencing (WGS): seven samples from Victoria, four from NSW and four from WA. All four ribotype 244 strains held by the UK-based CDRN reference laboratory (three North American isolates (from New Jersey, 2004; Indiana, 2011; 2007) and a recent UK clinical isolate from November 2011) were also sequenced. Following subculture of a single colony on Columbia blood agar for $48 \mathrm{~h}$, DNA was extracted using a commercial kit (QIAamp, Qiagen, Hilden, Germany). A combination of standard Illumina and adapted protocols was used to produce multiplexed paired-end libraries. Pools of eight samples were sequenced at the Wellcome Trust Centre for Human Genetics, Oxford, UK, on the Illumina MiSeq platform (Illumina Inc., San Diego, CA), generating $150 \mathrm{bp}$ paired-end reads. The sequences reported in this paper have been deposited in the National Center for Biotechnology Information Sequence Read Archive under BioProject accession number PRJNA277962 and are available at http://www. ncbi.nlm.nih.gov/bioproject/277962.

Sequence reads were analysed and assembled using a pipeline developed specifically for bacterial genomes: Each isolate was mapped using Stampy v1.0.11 (without Burrows-Wheeler Aligner pre-mapping, using an expected substitution rate of 0.01) [25] to the $C$. difficile 630 reference genome (GenBank: AM180355.1), CD630 [26]. Single nucleotide variants (SNVs) were identified across all mapped non-repetitive core genome sites using the SAMtools (version 0.1.12-10) mpileup command with the extended base-alignment quality flag, after parameter tuning based on bacterial sequences. A consensus of at least $75 \%$ was required to support a SNV, and calls were required to be homozygous under a diploid model. Only SNVs supported by at least five reads, including one in each direction, were accepted. Sequence reads were also assembled de novo using Velvet [27], run with the Velvet Optimiser. De novo assemblies were used to determine in silico multilocus sequence types (MLST).

Sequences from the ribotype 244 isolates were compared with available fully-sequenced reference isolates, with sequences from a reference laboratory collection (CDRN), and a collection of clinical isolates from Oxfordshire, UK, representing all five $C$. difficile clades [28]. As ribotype 244 has been shown to be from Clade 2 [14], we included previously sequenced Clade 2 strains from the Oxfordshire clinical collection, including example ST1 strains (ribotype 027) and all available ST41, ST47, ST67, ST97 and ST114 strains.

Maximum likelihood phylogenetic trees were drawn based on variable sites called across all sequences, using PhyML [29] with a Hasegawa, Kishino and Yano (HKY) substitution model. Ribotype 244 sequences were analysed using BEAST 1.7.5 [30] to generate a
FIGURE 1

Quarterly hospital-identified CDI case numbers by

hospital type, Western Australia, 2010-2012 ( $=2,061)$

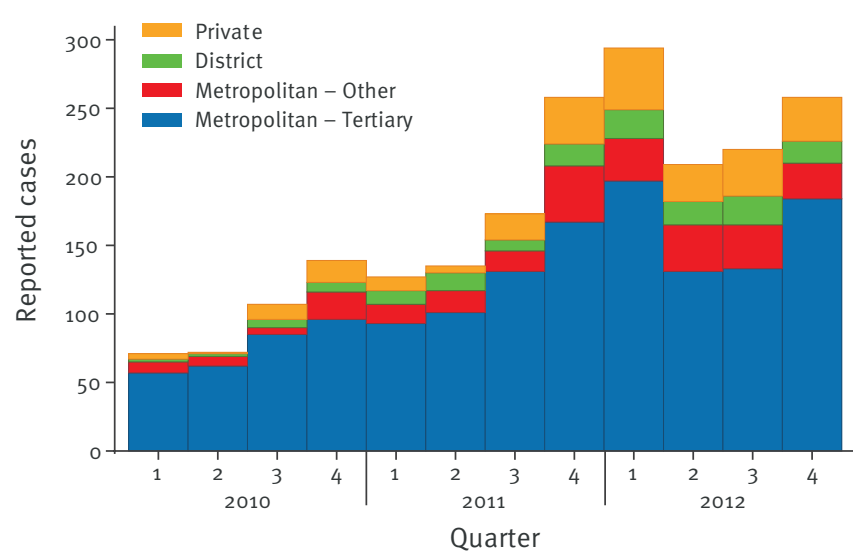

CDI: Clostridium difficile infection.

CDI was reported for patients attending any area of a hospital, i.e. all inpatient, emergency and outpatient departments. As such, reporting reflected the burden of CDI on a hospital and the surrounding community, as the reported cases included healthcare and community onset disease.

time-scaled phylogenetic tree and estimate the date of the most recent common ancestor of the outbreak strains. After identifying variable sites between the sequences, any variants clustered within 323 sites (the mean recombination insert size for Clade 2 isolates in a previous analysis [28]) were masked to remove these likely recombination events. To reduce the time for computation, any uncalled bases at otherwise invariant sites were assumed to be the same as the reference. We assumed a constant population size and a constant molecular clock at a previously estimated rate of $3.2 \times 10^{-7}$ substitutions per site per year [28]. Data from two convergent chains, initiated from different starting values, each of $10,000,000$ iterations, were combined after discarding the first 100,000 iterations as burn-in. A HKY substitution model was used with empiric base frequencies.

\section{Results}

\section{Epidemiology of Clostridium difficile infection in Western Australia}

During the three years of surveillance from January 2010 to December 2012 in Western Australia, there were a total of 2,061 hospital-identified CDI cases with a peak of 294 cases in quarter 12012 (Figure 1). Of 1,681 cases reported by metropolitan hospitals (where data on recent healthcare facility exposure was recorded), 1,086 (65\%) cases were HCF-associated, 478 (28\%) community-associated and 117 (7\%) indeterminate/unknown. Overall HCF-associated CDI incidence increased markedly in mid-2011 from 2.5/10,000 bed-days in the second quarter (April-June) to a peak at over 4.5/10,000 bed-days in the first quarter of 2012 (January-March) before declining over the next two 


\section{FIGURE 2}

Quarterly healthcare facility-associated and communityassociated CDI rates in metropolitan hospitals, Western Australia, 2010-2012 ( $\mathrm{n}=1,681)$

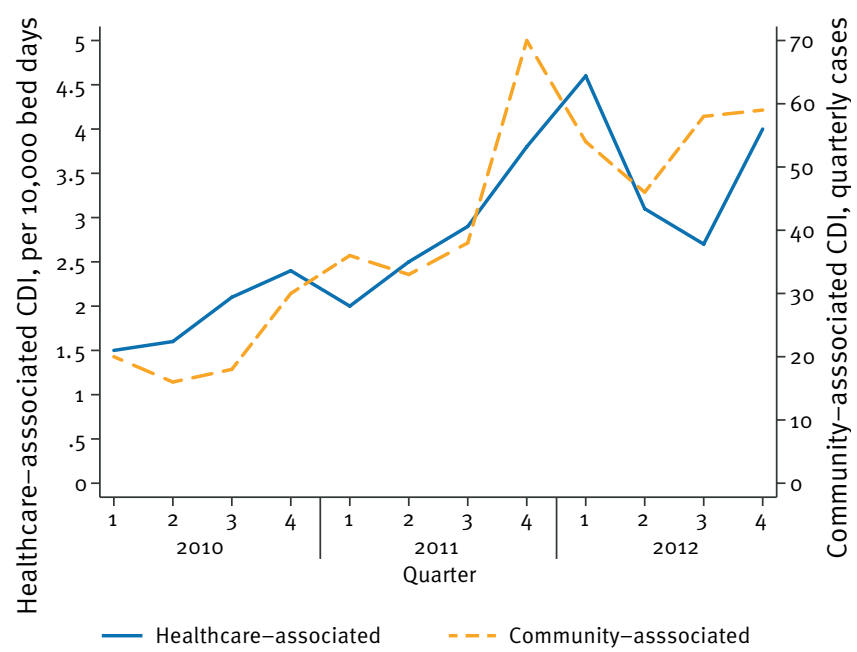

CDI: Clostridium difficile infection.

Rates are based on 1,681 (82\%) cases identified at metropolitan hospitals where data on recent healthcare exposure was available. The remaining 380 cases ( $18 \%$ of total 2,061 cases) were identified in smaller district and private hospitals where data on recent healthcare exposure was incomplete. The bedday denominator is based on bed-days in metropolitan hospitals only.

\section{TABLE}

Ribotypes causing CDI in Western Australia, October 2011-September $2012(\mathrm{n}=657)$

\begin{tabular}{|c|c|c|}
\hline Ribotype & Number of isolates & Prevalence \\
\hline 014/020 group & 264 & $35 \%$ \\
\hline 002 & 84 & $11 \%$ \\
\hline 056 & 40 & $5 \%$ \\
\hline 054 & 35 & $5 \%$ \\
\hline 052 & 32 & $4 \%$ \\
\hline 070 & 28 & $4 \%$ \\
\hline 015 & 24 & $3 \%$ \\
\hline 010 & 21 & $3 \%$ \\
\hline 244 & 21 & $3 \%$ \\
\hline 046 & 19 & $3 \%$ \\
\hline 017 & 19 & $3 \%$ \\
\hline QX001 & 18 & $2 \%$ \\
\hline 005 & 18 & $2 \%$ \\
\hline 064 & 17 & $2 \%$ \\
\hline QXo77 & 17 & $2 \%$ \\
\hline Total & 657 & $88 \%$ \\
\hline
\end{tabular}

CDI: Clostridium difficile infection.

Ribotypes were available for 746 CDI cases. The 15 most common ribotypes are shown, accounting for $88 \%$ of all ribotyped cases. Ribotype 244 accounted for 3\% of all cases. QX001 and QX077 were novel ribotypes. quarters, and then increasing again in the last quarter of 2012 (Figure 2). Trends in community-associated CDI rates followed the rates of healthcare-associated cases closely.

\section{Ribotype 244 epidemiology in Western \\ Australia}

In WA, between January 2010 and December 2012, 25 cases of ribotype 244 CDI were identified. During the main period of the outbreak, between 17 August 2011 and 23 April 2012, there were 19 cases of ribotype 244 CDI, with cases in each month except March. No further cases were recorded for over four months, until late August 2012, with six additional cases ( $\geq 1 /$ month) up to December 2012 (Figure 3). Before August 2011, no ribotype 244 isolate had been obtained from a CDI case in WA since ribotyping of cases began as part of comprehensive surveillance in January 2010. Only five cases were identified in WA in 2013, and no ribotype 244 cases have been identified in the first five months of 2014 .

The Table provides a breakdown of the 15 most commonly isolated ribotypes in WA in the first year for which complete ribotyping data were available from all hospitals, October 2011 to September 2012. Although ribotype 244 only accounted for $3 \%$ of cases, it was the ninth most commonly occurring ribotype, which represents a significant emergence of a novel ribotype.

Of the 25 ribotype 244 cases, 13 were female, 14 were 65 years or older, 14 presented at hospital emergency departments or were outpatients, and 14 presented at non-tertiary or community hospitals. Using surveillance definitions, 18 were community-onset (14 were community-associated infections and four were both community-onset and H(FA), and seven were both

\section{FIGURE 3}

Monthly ribotype 244 cases in Western Australia, 2011$2012(\mathrm{n}=25)$

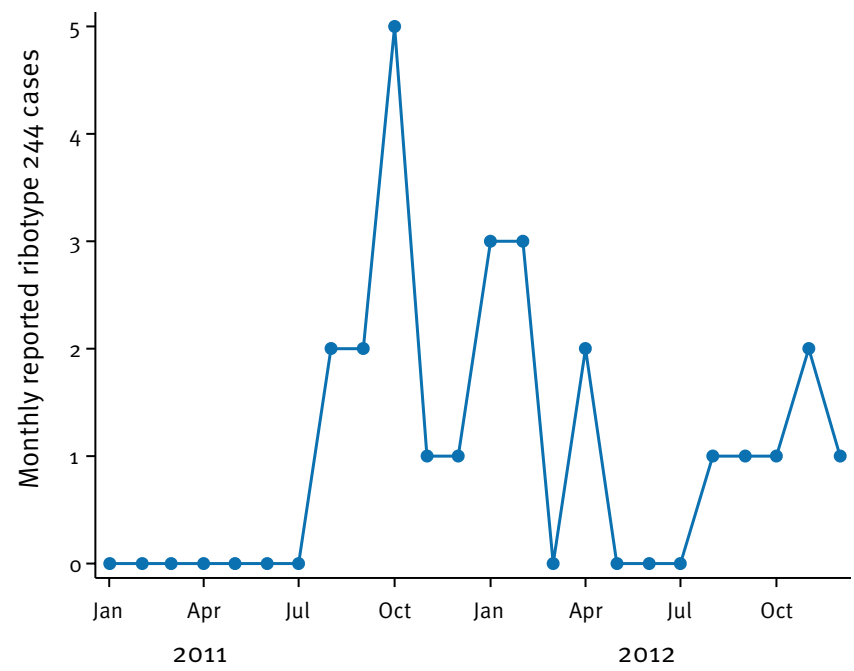


Phylogenetic relationship of outbreak ribotype 244 lineage, Western Australia, October 2011-September 2012, to global Clostridium difficile diversity

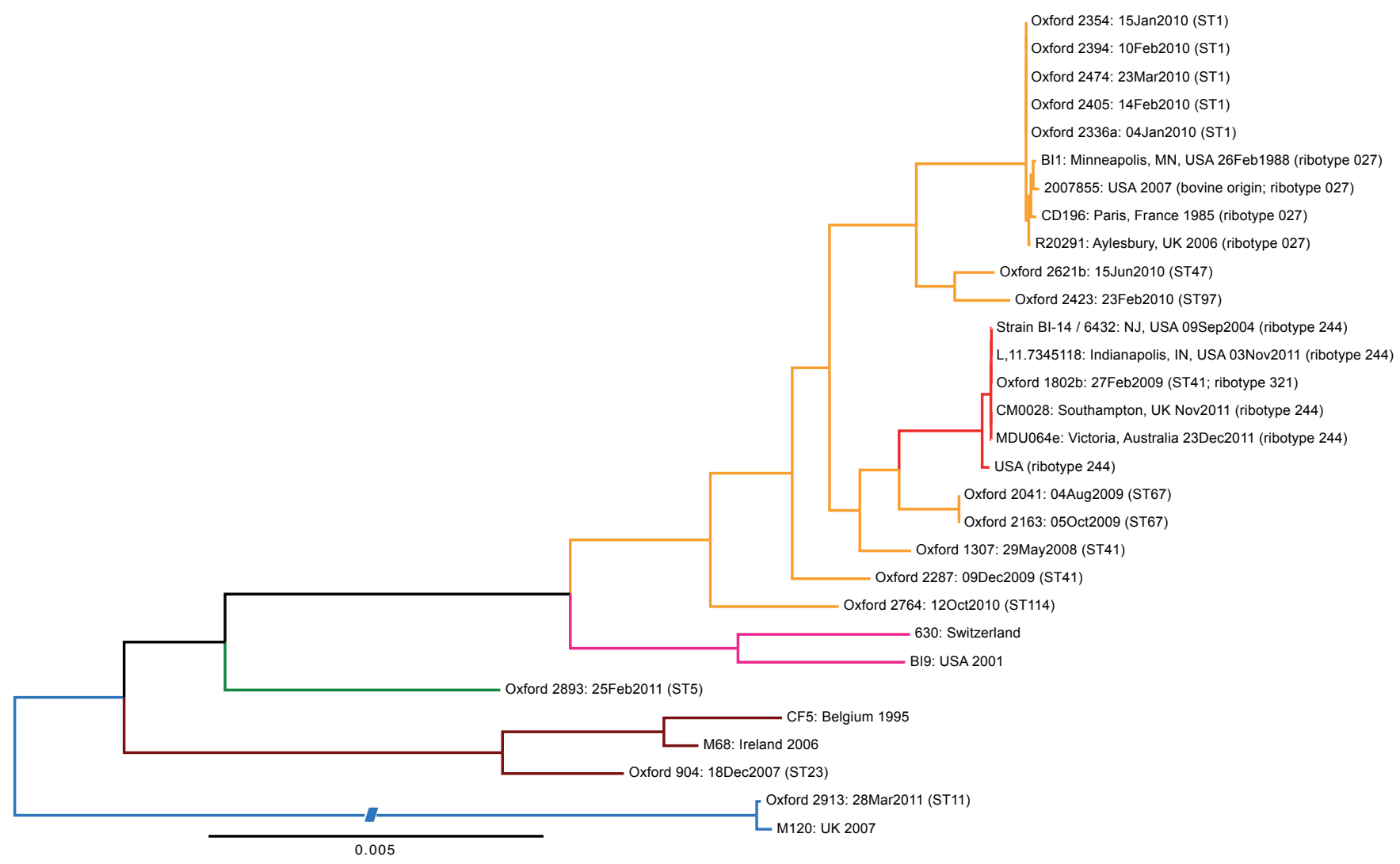

Reference isolates from the $C$. difficile clades are shown. One example ribotype 244 outbreak strain, MDU-064e, is included, with four UK reference collection ribotype 244 isolates. Example clinical strains from each of the five clades from an Oxfordshire, UK collection are shown, as well as five example strains from ST1/ribotype 027/NAP1, and all available samples from other Clade 2 STs.

Pink: Clade 1; orange: Clade 2 (outbreak lineage, and a closely related Oxfordshire ST41, ribotype 321, sample in red); green: Clade 3; maroon: Clade 4 ; blue: Clade 5 . Where available the date and location of isolation is shown.

Maximum likelihood tree based on 112,792 variable sites called across all sequences. Scale shows the number of substitutions per site, based on a whole genome length of $4290252 \mathrm{nt}$. The blue line depicting Clade 5 contains a break; the overall length of this branch is 0.028 .

HCF-onset and HCF-associated. The median patient age was 68 years (interquartile range: 38-88 years). In WA between January 2010 and December 2012, the proportion of ribotype 244 cases that were communityassociated, 14 of 25 (56\%), was significantly greater than the proportion of non-ribotype 244 cases with available data on healthcare exposure, 464 of 1,656 $(28 \%, p=0.006)$. No ward-based clustering of ribotype 244 HCF-associated cases was observed. Clinical data were available on 15 of the 25 ribotype 244 cases. Six cases had severe CDI as defined [18], but there were no deaths attributable to CDI.

All ribotype 244 isolates were positive for $t c d A, \operatorname{tcd} B$ and binary toxin and had a single nt deletion at position 117 in $t c d C$ seen in ribotype 027, but had no other deletions. All were susceptible to metronidazole, vancomycin, clindamycin and moxifloxacin, with modal minimum inhibitory concentrations of $0.5 \mathrm{mg} / \mathrm{L}, 2 \mathrm{mg} / \mathrm{L}$, $4 \mathrm{mg} / \mathrm{L}$ and $2 \mathrm{mg} / \mathrm{L}$, respectively. We determined the in silico multilocus sequence type (ST) of the ribotype 244 strains as ST41.

\section{Ribotype 244 evolution}

We investigated the phylogenetic relationship between whole genome sequences obtained from 15 Australian ribotype 244 outbreak isolates, four ribotype 244 isolates held by a UK reference laboratory, and existing sequences obtained from the five major $C$. difficile clades (Figure 4). In keeping with a previous study showing 10,803 SNVs between ribotype 244 and ribotype 027 isolates [14], we demonstrate the ribotype 244 lineage diverged relatively early from other Clade 2 lineages. It only shares a relatively ancient common ancestor with ST1 (ribotype 027). For example, we observed 12,026 SNVs between CD196 (a published ribotype 027 reference) and outbreak strain MDU-064e. The observed SNVs differ from the earlier comparison by Lim et al. because of the different ribotype 027 strains studied and the different 


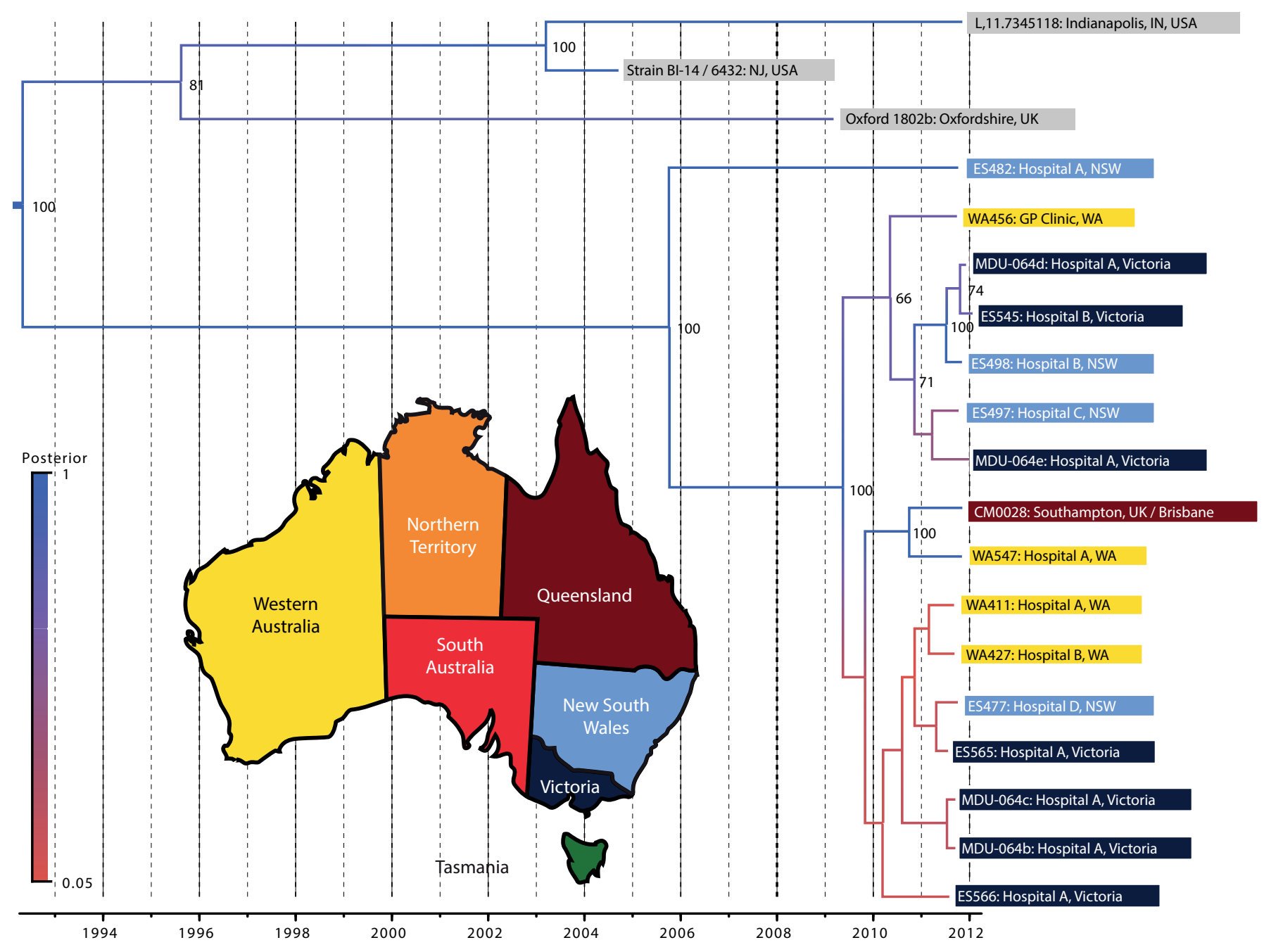

Tips are positioned by sampling date and coloured by Australian state of origin; grey: related UK and US strains; maroon: a closely related UK strain, as the patient had recently returned from Brisbane, Queensland. Tree branches are coloured by the posterior clade probability for each node. As indicated in the legend, blue branches are strongly supported by the sequence data and red branches more weakly supported. Nodes with posterior probabilities greater than $50 \%$ are annotated with the posterior support as a percentage. The terminal branches with low support at the bottom of the tree indicate that these isolates are similar (within four observed SNVs), consistent with a point source, but the exact ordering of their ancestry is not certain from the data.

sequence assembly and analysis approaches used. It is likely that, despite both belonging to the same clade, the current ribotype 244 is separated from ribotype 027 by hundreds/thousands of years given rates of C. difficile evolution $[5,28,31]$.

\section{Ribotype 244 outbreak phylogeny}

All 15 Australian ribotype 244 isolates were within 16 observed SNVs of each other, and all but one isolate were within eight SNVs, including a cluster of seven cases from three different states which were within four SNVs of each other. We compared the phylogenetic relationship between the outbreak isolates using a time-scaled Bayesian phylogeny (Figure 5). Excluding the single outlying isolate, the estimated date of the most recent common ancestor of the outbreak isolates was April 2009 (95\% credibility interval: March
2008-April 2010), and including all Australian ribotype 244 isolates, it was August 2005 (95\% credibility interval: December 2002-February 2008). Despite sequenced isolates originating from Australian towns and cities separated by thousands of kilometres, the isolates were closely genetically related, and without evidence of geographical intra-state clustering of similar isolates.

Interestingly, a ribotype 244 isolate held in the CDRN reference laboratory, from a patient in Southampton, UK, fell within the diversity of the Australian outbreak strains. Further epidemiological investigation initiated as a result of the sequencing data revealed that the UK patient had returned from Australia (Brisbane, Queensland) three weeks before the onset of diarrhoeal symptoms; this person had spent ca three 
months in Australia in late 2011. The patient had not made any visits to hospitals or GPs while in Australia nor stayed with anyone who was ill or on antibiotics. One relative they visited worked in a care home. The patient remained well until they required antibiotics for an unrelated complaint after returning to the UK. They developed diarrhoea shortly thereafter and were diagnosed with CDI within three weeks of return to the UK. The only risk factors for CDI identified in the UK patient were age over 65 years and recent antibiotic use.

Two ribotype 244 isolates from the US, one previously identified as ribotype 244 in [32], and a ribotype 321 isolate from Oxfordshire, UK were closely related to, but distinct from, the Australian ribotype 244 outbreak isolates (Figure 5). There are no reports of ribotype 321 in Australia. Only two ribotype 321 isolates have been identified by the UK CDRN reference laboratory to date, the Oxfordshire clinical strain and a food research centre isolate from Ireland in 2010.

\section{Discussion}

Here we describe a clonal outbreak of ribotype 244 throughout Australia, against a background of rising CDI incidence. Isolates originating from four states across Australia were genetically closely related without any evidence of geographical or temporal structure in their phylogenetic relationships, and over half of cases occurred without recent healthcare exposure, suggesting a possible community-based point source dispersed nationally. The majority of ribotype 244 cases were community-onset and many were detected in patientsyounger than 65 years and in those who presented to hospital emergency departments, rather than in hospital inpatients. Notably, by using the discriminatory power of WGS, we were also able to identify spread of the outbreak strain to the UK via a returning traveller. Since the end of 2012 there has been a decline in new cases of ribotype 244 in WA, supporting the suggestion that the cases in 2011/12 represented an outbreak followed by some ongoing transmission.

In contrast to Europe and North America, Australia has not seen large epidemics with $C$. difficile ribotype 027 despite at least two introductions $[6,7]$; it is possible that relatively conservative antimicrobial prescribing practices [8] and/or the geographical isolation of Australia have been responsible [33]. Despite the limited numbers of ribotype 244 cases seen, this outbreak does demonstrate that there is potential for epidemic spread in Australia too. Although ribotype 244 only accounted for $3 \%$ of all cases in WA, the outbreak occurred against a background of rising CDI incidence. Since mandatory reporting was introduced three years ago, rates of CDI in WA have more than doubled (Figure 2). Australia-wide increases in CDI incidence could be due to a number of factors. Increased awareness of CDI may have increased testing [34]; however, a $25 \%$ increase in laboratory testing in WA is very unlikely to account for the observed rise in CDI cases. Similarly, more sensitive testing methods could have resulted in increased reported incidence, with many laboratories changing from enzyme immunoassay testing, with ca $60-85 \%$ sensitivity, to nucleic acid amplification tests, with more than $95 \%$ sensitivity (but decreased specificity) [35]. Changes in laboratory testing during late $\mathbf{2 0 1 0}$ may have accounted for some of the increase in measured $\mathrm{CDI}$ rates in WA in quarters 3 and 4 of 2010. However, such potential laboratory ascertainment bias does not explain the increases in CDI rates recorded from quarter 3 onwards in 2011.

The introduction of new $C$. difficile strains, including ribotype 244 , alongside rises in the incidence of established strains, underlies the observed increased incidence of CDI overall. Two snap-shot surveys from the Australian Commission on Safety and Quality in Healthcare, the first in October and November 2010, and the second in October and November 2012, described the ribotypes of $C$. difficile circulating in Australia. Representative samples of 330 and 556 isolates, respectively, predominantly from hospital laboratories, were collected from all Australian states and ribotyped. The most common ribotypes in 2010 were $014 / 020$ (30\%), followed by 002 (11\%); a ribotype could not be assigned to $53 \%$ of isolates. By 2012 , ribotypes $014 / 020$ and 002 still accounted for $25 \%$ and $10 \%$ of CDI, respectively, but significant increases in the proportion of cases due to ribotypes 056, 070, 054, 015, 017, 053 and 244 were seen compared with 2010, with each of these ribotypes accounting for between $3 \%$ and $6 \%$ of cases (data not shown).

There now appears to be seasonality to CDI incidence in Australia; this has been demonstrated in other countries [36], but has been assumed to be due to healthcare-associated CDI following increased antibiotic treatment of winter-associated severe respiratory tract infections. By contrast, much of the increase in Australian CDI incidence has been due to communityassociated CDI cases with no previous contact with the hospital system $[12,13]$. The most likely explanation for this is the combination of community-associated strains, and the seasonal peak in antimicrobial prescriptions in the community, following respiratory tract infections [37].

The detection of ribotype 244 in Australia is a recent occurrence. Retrospectively, only one of the unidentified isolates (from Queensland) from the 2010 snapshot survey was found to be ribotype 244 . In addition to our data showing the emergence of ribotype 244 in WA where it had not been previously detected, recent surveys by other researchers suggest it is also present in other states. In a point prevalence survey in April 2012, 83 CDI isolates from Brisbane, Queensland were ribotyped and seven (8\%) of them were ribotype 244 (personal communication, David Patterson, July 2013). Between June 2011 and August 2013, ribotype 244 accounted for 56 of 3,111 ( $2 \%$ ) CDI cases in the North Sydney healthcare region. No ribotype 244 cases were seen in the region after January 2013 (data not shown), in keeping with the decline in ribotype 244 cases seen 
in WA from 2013 onwards. A limitation of surveillance data used in our study and the surveys described above, is that they were hospital-based. Although cases tested as outpatients were included, patients tested by general practitioners working outside of hospitals were not. As such the surveillance may underestimate the proportion of total CDI accounted for by strains predominantly causing community-associated disease.

The recent emergence of ribotype 244 in surveillance data is in keeping with our estimated date (2009) for the most recent common ancestor of the majority of the outbreak strains. If a point source was the explanation for the outbreak we might have expected the most recent common ancestor to be closer in time to the beginning of the outbreak, i.e. around 2011, and fewer than the observed eight SNVs separating the majority of the outbreak strains. However it is also possible that some within-host/within-reservoir diversity existed in the source of the outbreak before the first cases, analogous to the within-host diversity seen in some CDI $[28,38]$. A single strain from NSW was more diverse, and may represent a separate introduction, or pre-existing diversity in the source of the outbreak.

Although ribotype 244 strains share some features with ribotype 027 , WGS suggests these strains are substantially different at a whole genome level [14], confirmed in this study and in keeping with a previous description based on microarray data [39]. Ribotype 244 strains produce toxins A, B and binary toxin, are susceptible to fluoroquinolones and associated with more severe disease $[14,15]$.

We have demonstrated that ribotype 244 CDI was significantly more likely to be community-associated than other ribotypes, $56 \%$ of ribotype 244 cases were community-associated, compared with $28 \%$ of other ribotypes. In keeping with our data, there is also some evidence that cases from New Zealand were more likely to be community-associated than controls, $50 \%$ of cases vs $15 \%$ of controls $(p=0.08)$ [15]. In another case-control study in Victoria, Australia, proportionally more ribotype 244 cases were community-associated (four of 12) than non-ribotype 244 controls (six of 24), however this was not statistically significant $(p=0.72)[14]$. The relative excess of community-associated ribotype $244 \mathrm{CDI}$ suggests a possible community source or reservoir. The nature of this source is unknown but is unlikely to be spores shed from recently hospitalised patients following discharge as this strain is different from those circulating in Australian healthcare facilities (predominantly ribotypes 014/020 and 002). One potential reservoir of infection in the community is animals [40]. C. difficile is known to colonise or infect animals and outside of Australia, the predominant animal strain is ribotype 078 , commonly isolated from pigs and cattle [41]. This strain is responsible for increasing rates of community-acquired CDI in Europe $[42,43]$, suggesting that a zoonosis exists, however transmission of ribotype 244 from animals has not been observed to date. Contaminated food represents another potential source. C. difficile contamination of food has been previously reported in retail meat products in the US $[44,45]$, in ready-to-eat salads/vegetables in Scotland [46] and France [47], and in vegetables and seafood in Canada [48,49], but again ribotype 244 has not been isolated from food to date. The recent emergence of ribotype 244 in Australia suggests it may have been imported. The oldest ribotype 244 isolate in our collection, from 2004, was from New Jersey, US, suggesting the current outbreak may have originated from North America, however this may represent sampling bias only.

The numbers of ribotype 244 cases being seen in Australia currently, although high compared with previous surveys, do not completely account for the increased CDI rates seen over the past two years. Ribotypes circulating previously account for many cases, e.g. 014/020, 002, 056, and 054 (Table). However other new ribotypes of $C$. difficile may have been introduced into the country recently or emerged locally. We already have preliminary evidence of this in a significant number of infections with ribotype 251 C. difficile, another strain that has not been found in Australia previously (data not shown). Our data demonstrate that new strains causing CDI are an ongoing threat in Australia and worldwide, with rapid potential for spread as has been seen with ribotype 027 [5]. Active surveillance of CDI cases [50,51] alongside strain typing and rapid sequencing [52] is needed to identify and control emerging lineages.

\section{Acknowledgements}

This study was supported by the NIHR Oxford Biomedical Research Centre and the UKCRC Modernising Medical Microbiology Consortium, the latter funded under the UKCRC Translational Infection Research Initiative supported by Medical Research Council, Biotechnology and Biological Sciences Research Council and the National Institute for Health Research on behalf of the Department of Health (Grant G0800778) and the Wellcome Trust (Grant 087646/Z/08/Z). We acknowledge the support of Wellcome Trust core funding (Grant 090532/Z/09/Z). TEAP and DWC are NIHR Senior Investigators. DWE is a NIHR Doctoral Research Fellow.

\section{Conflict of interest}

The institution of DWC and TEAP received per-case funding from Optimer Pharmaceuticals to support fidaxomicin trial patient expenses. DWC and TEAP also received honoraria from Optimer Pharmaceuticals for participation in additional meetings related to investigative planning for fidaxomicin. MHW has received honoraria for consultancy work, financial support to attend meetings and research funding from Actelion, Alere, Astellas, Astra-Zeneca, bioMerieux, Cerexa, Durata, Cubist, Nabriva, Novacta, Pfizer, Roche, SanofiPasteur, Summit, The Medicines Company and VH Squared. No other author has a conflict of interest. 


\section{Authors' contributions}

DWE, LT, ASW, TEAP, DWC, MHW and TVR designed the study. DWE, LT, BE, CS, PGH, RLS, TMK, GK, RM, DG, WNF, PA, KED gathered the data. DWE, LT, BE, ASW, TEAP, TVR analysed the data. DWE and TVR wrote the first draft. All authors revised the manuscript and decided to publish. All authors vouch for the completeness and accuracy of the data and analysis.

\section{References}

1. Magill SS, Edwards JR, Bamberg W, Beldavs ZG, Dumyati G, Kainer MA, et al.; Emerging Infections Program HealthcareAssociated Infections and Antimicrobial Use Prevalence Survey Team. Multistate point-prevalence survey of health careassociated infections. N Engl J Med. 2014;370(13):1198-208. http://dx.doi.org/10.1056/NEJMoa1306801 PMID:24670166

2. McGlone SM, Bailey RR, Zimmer SM, Popovich MJ, Tian Y, Ufberg $\mathrm{P}$, et al. The economic burden of Clostridium difficile. Clin Microbiol Infect. 2012;18(3):282-9. http://dx.doi. org/10.1111/j.1469-0691.2011.03571.x PMID:21668576

3. Jernberg C, Löfmark S, Edlund C, Jansson JK. Long-term impacts of antibiotic exposure on the human intestinal microbiota. Microbiology. 2010;156(Pt 11):3216-23. http:// dx.doi.org/10.1099/mic.0.040618-o PMID:20705661

4. Wilcox MH, Mooney L, Bendall R, Settle CD, Fawley WN. A casecontrol study of community-associated Clostridium difficile infection. J Antimicrob Chemother. 2008;62(2):388-96. http:// dx.doi.org/10.1093/jac/dkn163 PMID:18434341

5. He M, Miyajima F, Roberts P, Ellison L, Pickard DJ, Martin MJ, et al. Emergence and global spread of epidemic healthcareassociated Clostridium difficile. Nat Genet. 2013;45(1):109-13. PMID:23222960

6. Riley TV, Thean S, Hool G, Golledge CL. First Australian isolation of epidemic Clostridium difficile PCR ribotype 027. Med J Aust. 2009;190(12):706-8. PMID:19527210

7. Richards M, Knox J, Elliott B, Mackin K, Lyras D, Waring LJ, et al. Severe infection with Clostridium difficile PCR ribotype 027 acquired in Melbourne, Australia. Med J Aust. 2011;194(7):36971. PMID:21470090

8. Cheng AC, Turnidge J, Collignon P, Looke D, Barton M, Gottlieb T. Control of fluoroquinolone resistance through successful regulation, Australia. Emerg Infect Dis. 2012;18(9):1453-60. http://dx.doi.org/10.3201/eid1809.111515 PMID:22932272

9. Khanna S, Pardi DS, Aronson SL, Kammer PP, Orenstein R, St Sauver JL, et al. The epidemiology of community-acquired Clostridium difficile infection: a population-based study. Am J Gastroenterol. 2012;107(1):89-95. http://dx.doi.org/10.1038/ ajg.2011.398 PMID:22108454

10. Health Protection Agency (HPA). Quarterly Epidemiological Commentary: Mandatory MRSA bacteraemia, Clostridium difficile infection \& MSSA bacteraemia (up to January - March 2012). London: HPA; 2012.

11. Viseur N, Lambert M, Delmée M, Van Broeck J, Catry B. Nosocomial and non-nosocomial Clostridium difficile infections in hospitalised patients in Belgium: compulsory surveillance data from 2008 to 2010. Euro Surveill. 2011;16(43): pii=20000. PMID:22085603

12. Slimings C, Armstrong P, Beckingham WD, Bull AL, Hall L, Kennedy KJ, et al. Increasing incidence of Clostridium difficile infection, Australia, 2011-2012. Med J Aust. 2014;200(5):272-6. http://dx.doi.org/10.5694/mja13.11153 PMID:24641152

13. Mitchell BG, Wilson F, McGregor A. An increase in community onset Clostridium difficile infection: a population-based study, Tasmania, Australia. Healthcare Infection. 2012;17(4):127-132. http://dx.doi.org/10.1071/Hl12029

14. Lim SK, Stuart RL, Mackin KE, Carter GP, Kotsanas D, Francis MJ, et al. Emergence of a ribotype 244 strain of Clostridium difficile associated with severe disease and related to the epidemic ribotype 027 strain. Clin Infect Dis. 2014;58(12):1723 30. http://dx.doi.org/10.1093/cid/ciu203 PMID:24704722

15. De Almeida MN, Heffernan H, Dervan A, Bakker S, Freeman JT, Bhally $\mathrm{H}$, et al. Severe Clostridium difficile infection in New Zealand associated with an emerging strain, PCR-ribotype 244 . N Z Med J. 2013;126(1380):9-14. PMID:24126745

16. Kuijper EJ, Coignard B, Tüll P, ESCMID Study Group for Clostridium difficile, EU Member States, European Centre for Disease Prevention and Control. Emergence of Clostridium difficile-associated disease in North America and Europe. Clin Microbiol Infect. 2006;12(s6) Suppl 6;2-18. http://dx.doi. org/10.1111/j.1469-0691.2006.01580.x PMID:16965399

17. Cohen SH, Gerding DN, Johnson S, Kelly CP, Loo VG, McDonald LC, et al.; Society for Healthcare Epidemiology of America;
Infectious Diseases Society of America. Clinical practice guidelines for Clostridium difficile infection in adults: 2010 update by the society for healthcare epidemiology of America (SHEA) and the infectious diseases society of America (IDSA). Infect Control Hosp Epidemiol. 2010;31(5):431-55. http:// dx.doi.org/10.1086/651706 PMID:20307191

18. Bauer MP, Kuijper EJ, van Dissel JT; European Society of Clinical Microbiology and Infectious Diseases. European Society of Clinical Microbiology and Infectious Diseases (ESCMID): treatment guidance document for Clostridium difficile infection (CDI). Clin Microbiol Infect. 2009;15(12):1067-79. http://dx.doi. org/10.1111/j.1469-0691.2009.03099.x PMID:19929973

19. Kato N, Ou CY, Kato H, Bartley SL, Brown VK, Dowell VR Jr, et al. Identification of toxigenic Clostridium difficile by the polymerase chain reaction. J Clin Microbiol. 1991;29(1):33-7. PMID:1993763

20. Stubbs S, Rupnik M, Gibert M, Brazier J, Duerden B, Popoff M. Production of actin-specific ADP-ribosyltransferase (binary toxin) by strains of Clostridium difficile. FEMS Microbiol Lett. 2000;186(2):307-12. http://dx.doi. org/10.1111/j.1574-6968.2000.tb09122.x PMID:10802189

21. Kato H, Kato N, Watanabe K, Iwai N, Nakamura H, Yamamoto $\mathrm{T}$, et al. Identification of toxin A-negative, toxin B-positive Clostridium difficile by PCR. J Clin Microbiol. 1998;36(8):217882. PMID:9665986

22. Stubbs SL, Brazier JS, O’Neill GL, Duerden BI. PCR targeted to the 16 S-23S rRNA gene intergenic spacer region of Clostridium difficile and construction of a library consisting of 116 different PCR ribotypes. J Clin Microbiol. 1999;37(2):461-3. PMID:9889244

23. Knetsch CW, Lawley TD, Hensgens MP, Corver J, Wilcox MW, Kuijper EJ. Current application and future perspectives of molecular typing methods to study Clostridium difficile infections. Euro Surveill. 2013;18(4):20381. PMID:23369393

24. Clinical and Laboratory Standards Institute (CLSI). Methods for antimicrobial susceptibility testing of anaerobic bacteria; approved standard - eighth edition. CLSI document M11-A8. Wayne: CLSI; 2012

25. Lunter G, Goodson M. Stampy: a statistical algorithm for sensitive and fast mapping of Illumina sequence reads. Genome Res. 2011;21(6):936-9. http://dx.doi.org/10.1101/ gr.111120.110 PMID:20980556

26. Sebaihia M, Wren BW, Mullany P, Fairweather NF, Minton N, Stabler R, et al. The multidrug-resistant human pathogen Clostridium difficile has a highly mobile, mosaic genome. Nat Genet. 2006;38(7):779-86. http://dx.doi.org/10.1038/ng1830 PMID:16804543

27. Zerbino DR, Birney E. Velvet: algorithms for de novo short read assembly using de Bruijn graphs. Genome Res. 2008;18(5):821-9. http://dx.doi.org/10.1101/gr.074492.107 PMID:18349386

28. Didelot X, Eyre DW, Cule M, Ip CL, Ansari MA, Griffiths D, et al. Microevolutionary analysis of Clostridium difficile genomes to investigate transmission. Genome Biol. 2012;13(12):R118. http://dx.doi.org/10.1186/gb-2012-13-12-r118 PMID:23259504

29. Guindon S, Gascuel O. A simple, fast, and accurate algorithm to estimate large phylogenies by maximum likelihood. Syst Biol. 2003;52(5):696-704. http://dx.doi. org/10.1080/10635150390235520 PMID:14530136

30. Drummond AJ, Suchard MA, Xie D, Rambaut A. Bayesian phylogenetics with BEAUti and the BEAST 1.7. Mol Biol Evol. 2012;29(8):1969-73. http://dx.doi.org/10.1093/molbev/mss075 PMID:22367748

31. He M, Sebaihia M, Lawley TD, Stabler RA, Dawson LF, Martin MJ, et al. Evolutionary dynamics of Clostridium difficile over short and long time scales. Proc Natl Acad Sci USA. 2010;107(16):7527-32. http://dx.doi.org/10.1073/ pnas.0914322107 PMID:20368420

32. Valiente E, Dawson LF, Cairns MD, Stabler RA, Wren BW. Emergence of new $P C R$ ribotypes from the hypervirulent Clostridium difficile 027 lineage. J Med Microbiol. 2012;61(Pt 1):49-56. http://dx.doi.org/10.1099/jmm.0.036194-0 PMID:21903827

33. Clements ACA, Magalhães RJS, Tatem AJ, Paterson DL, Riley TV. Clostridium difficile PCR ribotype 027: assessing the risks of further worldwide spread. Lancet Infect Dis. 2010;10(6):395404. http://dx.doi.org/10.1016/S1473-3099(10)70080-3 PMID:20510280

34. Bauer MP, Notermans DW, van Benthem BH, Brazier IS, Wilcox MH, Rupnik M, et al.; ECDIS Study Group. Clostridium difficile infection in Europe: a hospital-based survey. Lancet. 2011;377(9759):63-73. http://dx.doi.org/10.1016/S01406736(10)61266-4 PMID:21084111

35. Planche TD, Davies KA, Coen PG, Finney JM, Monahan $I M$, Morris KA, et al. Differences in outcome according to Clostridium difficile testing method: a prospective multicentre 
diagnostic validation study of $C$. difficile infection. Lancet Infect Dis, 12013;3(11):936-945. http://dx.doi.org/10.1016/ S1473-3099(13)70200-7 PMID: 24007915

36. Polgreen PM, Yang M, Bohnett LC, Cavanaugh JE. A time-series analysis of clostridium difficile and its seasonal association with influenza. Infect Control Hosp Epidemiol. 2010;31(4):3827. http://dx.doi.org/10.1086/651095 PMID:20175682

37. Kwong JC, Maaten S, Upshur REG, Patrick DM, Marra $F$. The effect of universal influenza immunization on antibiotic prescriptions: an ecological study. Clin Infect Dis. 2009;49(5):750-6. http://dx.doi.org/10.1086/605087 PMID:19624280

38. Eyre DW, Walker AS, Freeman J, Baines SD, Fawley WN, Chilton $\mathrm{CH}$, et al. Short-term genome stability of serial Clostridium difficile ribotype 027 isolates in an experimental gut model and recurrent human disease. PLoS ONE. 2013;8(5):e63540. http://dx.doi.org/10.1371/journal.pone.0063540 PMID:23691061

39. Stabler RA, Gerding DN, Songer JG, Drudy D, Brazier JS, Trinh HT, et al. Comparative phylogenomics of Clostridium difficile reveals clade specificity and microevolution of hypervirulent strains. J Bacteriol. 2006;188(20):7297-305. http://dx.doi. org/10.1128/JB.00664-06 PMID:17015669

40. Hensgens MPM, Keessen EC, Squire MM, Riley TV, Koene MGJ, de Boer E, et al.; European Society of Clinical Microbiology and Infectious Diseases Study Group for Clostridium difficile (ESGCD). Clostridium difficile infection in the community: a zoonotic disease? Clin Microbiol Infect. 2012;18(7):63545. http://dx.doi.org/10.1111/j.1469-0691.2012.03853.x PMID:22536816

41. Squire MM, Riley TV. Clostridium difficile infection in humans and piglets: a 'One Health' opportunity. Curr Top Microbiol Immunol. 2013;365:299-314. http://dx.doi. org/10.1007/82_2012_237 PMID:22695920

42. Goorhuis A, Bakker D, Corver J, Debast SB, Harmanus C, Notermans DW, et al. Emergence of Clostridium difficile infection due to a new hypervirulent strain, polymerase chain reaction ribotype 078. Clin Infect Dis. 2008;47(9):1162-70. http://dx.doi.org/10.1086/592257 PMID:18808358

43. Burns K, Morris-Downes M, Fawley WN, Smyth E, Wilcox $\mathrm{MH}$, Fitzpatrick F. Infection due to $\mathrm{C}$. difficile ribotype 078: first report of cases in the Republic of Ireland. J Hosp Infect. 2010;75(4):287-91. http://dx.doi.org/10.1016/j. jhin.2010.03.025 PMID:20621236

44. Rodriguez-Palacios A, Reid-Smith RJ, Staempfli HR, Daignault D, Janecko N, Avery BP, et al. Possible seasonality of Clostridium difficile in retail meat, Canada. Emerg Infect Dis. 2009;15(5):802-5. http://dx.doi.org/10.3201/eid1505.081084 PMID:19402975

45. Songer JG, Trinh HT, Killgore GE, Thompson AD, McDonald LC, Limbago BM. Clostridium difficile in retail meat products, USA, 2007. Emerg Infect Dis. 2009;15(5):819-21. http://dx.doi. org/10.3201/eid1505.081071 PMID:19402980

46. Bakri MM, Brown DJ, Butcher IP, Sutherland AD. Clostridium difficile in ready-to-eat salads, Scotland. Emerg Infect Dis. 2009;15(5):817-8. http://dx.doi.org/10.3201/eid1505.081186 PMID:19402979

47. Eckert C, Burghoffer B, Barbut F. Contamination of ready-toeat raw vegetables with Clostridium difficile in France. J Med Microbiol. 2013;62(Pt 9):1435-8. http://dx.doi.org/10.1099/ jmm.0.056358-o PMID:23449876

48. Metcalf DS, Costa MC, Dew WMV, Weese JS. Clostridium difficile in vegetables, Canada. Lett Appl Microbiol. 2010;51(5):600-2. http://dx.doi.org/10.1111/j.1472765X.2010.02933.x PMID:21069911

49. Metcalf D, Avery BP, Janecko N, Matic N, Reid-Smith $R$, Weese JS. Clostridium difficile in seafood and fish. Anaerobe. 2011;17(2):85-6. http://dx.doi.org/10.1016/j. anaerobe.2011.02.008 PMID:21376822

50. Schlackow I, Walker AS, Dingle K, Griffiths D, Oakley S, Finney J, et al. Surveillance of infection severity: a registry study of laboratory diagnosed Clostridium difficile. PLoS Med. 2012;9(7):e1001279. http://dx.doi.org/10.1371/journal. pmed.1001279 PMID:22859914

51. Wilcox MH, Shetty N, Fawley WN, Shemko M, Coen P, Birtles A, et al. Changing epidemiology of Clostridium difficile infection following the introduction of a national ribotyping. based surveillance scheme in England. Clin Infect Dis. 2012;55(8):1056-63. http://dx.doi.org/10.1093/cid/cis614 PMID:22784871

52. Eyre DW, Golubchik T, Gordon NC, Bowden R, Piazza P, Batty $E M$, et al. A pilot study of rapid benchtop sequencing of Staphylococcus aureus and Clostridium difficile for outbreak detection and surveillance. BMJ Open. 2012;2(3):e001124. http://dx.doi.org/10.1136/bmjopen-2012-001124 PMID:22674929 